\title{
Burgers' Equations in the Riemannian Geometry Associated with First-Order Differential Equations
}

\author{
Z. Ok Bayrakdar $\mathbb{D}^{1}$ and T. Bayrakdar $\mathbb{D}^{2}$ \\ ${ }^{1}$ Department of Physics, Ege University, 35040 İzmir, Turkey \\ ${ }^{2}$ Department of Mathematics, Akdeniz University, 07058 Antalya, Turkey \\ Correspondence should be addressed to Z. Ok Bayrakdar; zahideok@gmail.com
}

Received 12 October 2017; Revised 13 December 2017; Accepted 21 December 2017; Published 8 February 2018

Academic Editor: Boris G. Konopelchenko

Copyright (c) 2018 Z. Ok Bayrakdar and T. Bayrakdar. This is an open access article distributed under the Creative Commons Attribution License, which permits unrestricted use, distribution, and reproduction in any medium, provided the original work is properly cited.

\begin{abstract}
We construct metric connection associated with a first-order differential equation by means of the generator set of a Pfaffian system on a submanifold of an appropriate first-order jet bundle. We firstly show that the inviscid and viscous Burgers' equations describe surfaces attached to an ODE of the form $d x / d t=u(t, x)$ with certain Gaussian curvatures. In the case of PDEs, we show that the scalar curvature of a three-dimensional manifold encoding a system of first-order PDEs is determined in terms of the integrability condition and the Gaussian curvatures of the surfaces corresponding to the integral curves of the vector fields which are annihilated by the contact form. We see that an integral manifold of any PDE defines intrinsically flat and totally geodesic submanifold.
\end{abstract}

\section{Introduction}

A simple dynamical transport phenomenon such as transport of a pollutant in a fluid, transportation of information, wave propagation, traffic flow, population density, chemical reactions, and gas dynamics is described by a certain first-order partial differential equation (PDE) of space and time variables, the so-called transport equation $[1,2]$. Among them, Burgers' equation may be the more intriguing one which serves as a prototype model for turbulent flows [3]. Many works in the literature are devoted to investigation of Lie symmetries and the exact solutions of Burgers' equation(s) and its generalizations [4-14]. Beyond its importance in mathematical physics, it appears also as a geodesic equation on diffeomorphism group of a circle with right invariant $L^{2}$ metric (see [15] and references therein). As we show in this paper, the identity for the Gaussian curvature of a surface associated with a first-order ordinary differential equation (ODE) in the first-order jet bundle is described by an inhomogeneous Burgers' equation:

$$
u_{t}+u u_{x}=-\int^{x} \mathscr{K}\left(t, x^{\prime}\right) d x^{\prime} .
$$

Geometric study of differential equations is a notable subject in differential geometry and dates back to Tresse, Lie, and Cartan [16-19]. A considerable number of works in the literature are devoted to study of problems such as linearization, existence of local coordinates in which an equation takes the special form, and theory of connections associated with differential equations [20-27]. In modern terminology, an ODE or a PDE is identified with an exterior differential system in an appropriate jet bundle and this approach leads to geometrization of differential equations. In this work, we basically deal with the first-order ODEs and PDEs in the context of Riemannian geometry and Riemannian structure defined on a submanifold corresponding to given equation enables one to give a geometric description of an interesting class of equations in mathematical physics.

With this regard, in this paper, we construct $\mathfrak{o}(2, \mathbb{R})$ and $\mathfrak{v}(3, \mathbb{R})$-valued connections from the exterior differential systems encoding first-order ODEs and PDEs on corresponding submanifolds in the appropriate jet bundles. As a new result, we firstly show that the Gaussian curvature of a surface corresponding to a first-order ODE in $J^{1}(\mathbb{R}, \mathbb{R})$ is described by nonlinear PDE (1). Accordingly, we show that the inviscid and viscous Burgers' equations and Korteweg-de 
Vries (KdV) equation can be seen as the equations for surfaces with certain Gaussian curvatures. Also, Riemannian metrics for spaces of constant curvature can be attached to some linear ODEs by solving certain inhomogeneous Burgers' equations. For a system of first-order PDEs, we show that an integral manifold of any system of first-order PDEs defines intrinsically flat and totally geodesic submanifold. We prove that the scalar curvature of three-dimensional Riemannian manifold obtained from a system of first-order PDEs is given by the sum of the integrability condition and the Gaussian curvatures of the surfaces corresponding to the characteristic equations. Accordingly, we show that, for all points on an integral submanifold of a flat manifold, sectional curvatures are described by a pair of inhomogeneous Burgers' equations.

\section{Preliminaries}

2.1. Basics of Riemannian Geometry in an Orthonormal Frame. In this subsection, we shall give the basic notions of the formulation of the Riemannian geometry in an orthonormal frame. We assume that all considerations are local and all objects are smooth. For details in the subject we refer to [2830].

Let $(\mathscr{M}, g)$ be a $n$ dimensional Riemannian manifold and let $U$ be a coordinate neighbourhood of $\mathscr{M}$. Let $\Gamma_{U}(T \mathscr{M})$ and $\Gamma_{U}\left(T^{*} \mathscr{M}\right)$ denote the set of sections of the tangent and cotangent bundles of $\mathscr{M}$ over $U$, respectively. For simplicity, let us denote $\Gamma_{U}$ by $\Gamma$. Let $e_{i} \in \Gamma(T \mathscr{M})$ and $\omega^{i} \in \Gamma\left(T^{*} \mathscr{M}\right)$ for $i=1, \ldots, n$. An orthonormal frame field $\left(e_{1}, \ldots, e_{n}\right)$ on $U$, which is dual to the coframe field $\left(\omega^{1}, \ldots, \omega^{n}\right)$, is defined with respect to symmetric section $g$ of $T^{*} \mathscr{M} \otimes T^{*} \mathscr{M}$ as

$$
g=\sum_{i} \omega^{i} \otimes \omega^{i}
$$

here $\omega^{i}\left(e_{j}\right)=\delta_{j}^{i} \cdot g$ is called the Riemannian metric on $U$. The matrix of 1-forms $\theta=\left(\theta_{k}^{j}\right)$, satisfying

$$
d \omega^{i}=-\theta_{j}^{i} \wedge \omega^{j}, \quad \theta_{j}^{i}=-\theta_{i}^{j},
$$

is called unique $\mathfrak{o}(n, \mathbb{R})$-valued torsion-free connection on $U$. From (3), one has $\theta_{j}^{i}=\gamma_{j k}^{i} \omega^{k}$ for some functions $\gamma_{j k}^{i}$. The coefficients $\gamma_{j k}^{i}$ are defined in terms of the structure functions $\left[e_{i}, e_{j}\right]=-c_{i j}^{k} e_{k}$ as

$$
\gamma_{j k}^{i}=\frac{1}{2}\left(c_{[j k]}^{i}+c_{[k i]}^{j}-c_{[i j]}^{k}\right) .
$$

Clearly one has $\gamma_{j k}^{i}=-\gamma_{i k}^{j}$. Exterior derivative of (3) gives $0=\left(d \theta_{j}^{i}+\theta_{k}^{i} \wedge \theta_{j}^{k}\right) \wedge \omega^{j}$. This implies that

$$
\Omega_{j}^{i}=d \theta_{j}^{i}+\theta_{k}^{i} \wedge \theta_{j}^{k}, \quad \Omega_{j}^{i}=-\Omega_{i}^{j} .
$$

$\Omega=\left(\Omega_{j}^{i}\right)$ is called curvature 2 -form or the Riemannian curvature tensor associated with the connection $\theta$ and it is written as

$$
\Omega_{j}^{i}=\sum_{k<l} R_{j k l}^{i} \omega^{k} \wedge \omega^{l}
$$

Riemannian curvature tensor has the symmetries

$$
R_{j k l}^{i}=-R_{i k l}^{j}, \quad R_{j k l}^{i}=-R_{j l k}^{i} .
$$

Ricci curvature and the scalar curvature are defined, respectively, by

$$
R_{j l}=R_{j k l}^{k}, \quad R=R_{j j} .
$$

The covariant derivative operator associated with the connection $\theta$ is defined by

$$
\nabla: \Gamma(T \mathscr{M}) \longrightarrow \Gamma\left(T^{*} \mathscr{M} \otimes T \mathscr{M}\right)
$$

with

$$
\nabla_{X} e_{j}=\theta_{j}^{i}(X) e_{i}, \quad X \in \Gamma(T \mathscr{M})
$$

2.2. Jet Bundle Formulation of the First-Order Equations. Consider a first-order ODE of the form

$$
\frac{d x}{d t}=u(t, x) .
$$

For the function $F(t, x, p)=p-u(t, x)$, (11) can be described geometrically as a surface in the first-order jet bundle by $F^{-1}(0)$. That is,

$$
\mathcal{\delta}_{0}=\left\{(t, x, p) \in J^{1}(\mathbb{R}, \mathbb{R}) \mid F(t, x, p)=0\right\},
$$

where $(t, x, p)$ denotes standard local coordinates on $J^{1}(\mathbb{R}, \mathbb{R})$. The first-order jet bundle of maps $\mathbb{R} \rightarrow \mathbb{R}$ is a fibered manifold defined by the space of all 1-jets of smooth sections of the trivial bundle $\pi: \mathbb{R} \times \mathbb{R} \rightarrow \mathbb{R}$. The 1-graph of a solution curve of differential equation (11) is a curve on $\mathcal{S}_{0}$, represented by a section $t \mapsto\left(t, x(t), x^{\prime}(t)\right)$, on which contact form $\omega^{2}=d x-u d t$ vanishes. The one-form $\omega^{2}$ is defined by the pullback of the canonical contact form $\vartheta=d x-p d t$ to $\mathcal{S}_{0}$, by natural inclusion. Accordingly, solutions of the exterior differential system

$$
\begin{aligned}
& \omega^{2}=0, \\
& \omega^{1} \neq 0,
\end{aligned}
$$

with $\omega^{1}=d t$, are in one-to-one correspondence with the solutions of (11). For the vector fields

$$
\begin{aligned}
& e_{1}=\frac{\partial}{\partial t}+u \frac{\partial}{\partial x} \\
& e_{2}=\frac{\partial}{\partial x}
\end{aligned}
$$

on $\mathcal{S}_{0}$, we have the relation $\omega^{i}\left(e_{j}\right)=\delta_{j}^{i}$ and 1-graph of a solution curve of (11) is an integral curve of the vector field $e_{1}$.

Similar to ODEs, any first-order PDE with one dependent and two independent variables, in the form

$$
F\left(t, x, u, u_{t}, u_{x}\right)=0,
$$


is described as a submanifold in the first-order jet bundle of maps $\mathbb{R}^{2} \rightarrow \mathbb{R}$. In the vicinity of a point, where $\partial F / \partial u_{x} \neq 0$, Implicit Function Theorem states that one can write (15) as

$$
u_{x}=\phi\left(t, x, u, u_{t}\right)
$$

If $u_{t}$ is known, say $u_{t}=f(t, x, u)$, then (16) may be written as an overdetermined system

$$
\begin{aligned}
& u_{t}=f(t, x, u) \\
& u_{x}=g(t, x, u),
\end{aligned}
$$

with $g(t, x, u)=\phi(t, x, u, f(t, x, u))$, such that $u_{t x}=u_{x t}$ must hold on an integral surface. The first-order jet bundle of maps $\mathbb{R}^{2} \rightarrow \mathbb{R}$ is a fibered manifold defined by the space of all 1-jets of smooth sections of the trivial bundle $\pi: \mathbb{R}^{2} \times \mathbb{R} \rightarrow \mathbb{R}^{2}$ of two independent and one dependent variables and denoted by $J^{1}\left(\mathbb{R}^{2}, \mathbb{R}\right)$. Let $(t, x, u, p, q)$ be the standard coordinates on $J^{1}\left(\mathbb{R}^{2}, \mathbb{R}\right)$. The 1-graph of a solution or an integral surface of (15) is represented by the section $u_{t}=p$ and $u_{x}=q$ on which the canonical contact form

$$
\theta=d u-p d t-q d x
$$

vanishes. That is, solutions of the exterior differential system

$$
\begin{array}{r}
\theta=0, \\
d t \wedge d x \neq 0,
\end{array}
$$

are in one-to-one correspondence with the solutions of (17).

Consider three-dimensional submanifold $\Sigma \hookrightarrow J^{1}\left(\mathbb{R}^{2}\right.$, $\mathbb{R})$ determined by the equations

$$
\begin{aligned}
& p=f(t, x, u), \\
& q=g(t, x, u) .
\end{aligned}
$$

Let us denote the pullback of $\theta$ to $\Sigma$ by $\omega^{3}=d u-f(t, x, u) d t-$ $g(t, x, u) d x$. In terms of the differential 1-forms

$$
\begin{aligned}
& \omega^{1}=d t, \\
& \omega^{2}=d x, \\
& \omega^{3}=d u-f d t-g d x,
\end{aligned}
$$

defined on $\Sigma$ with local coordinates $(t, x, u)$, and the inclusion map, an integral submanifold $\mathcal{S}$ of (15) is determined by $\iota$ : $\mathcal{S} \hookrightarrow \Sigma \subset J^{1}\left(\mathbb{R}^{2}, \mathbb{R}\right)$ such that

$$
\begin{array}{r}
\iota^{*}\left(\omega^{3}\right)=0, \\
\iota^{*}\left(\omega^{1} \wedge \omega^{2}\right) \neq 0 .
\end{array}
$$

Since the exterior derivative commutes with the pullback, $\iota^{*} d \omega^{3}=0$ holds also on this two-dimensional integral manifold. Thereof, the identity $\mathscr{I}=f_{x}-g_{t}+f_{u} g-g_{u} f$ must vanish identically on integral manifold $\delta$. Due to the
Frobenius Theorem, the integrability condition is described by

$$
\iota^{*}\left(d \omega^{3} \wedge \omega^{3}\right)=0
$$

It is suitable to note here that $\mathscr{I}=0$ does not necessarily hold identically on $\Sigma$.

The vector fields

$$
\begin{aligned}
& e_{1}=\frac{\partial}{\partial t}+f \frac{\partial}{\partial u} \\
& e_{2}=\frac{\partial}{\partial x}+g \frac{\partial}{\partial u} \\
& e_{3}=\frac{\partial}{\partial u}
\end{aligned}
$$

satisfy the relation $\omega^{i}\left(e_{j}\right)=\delta_{j}^{i}$. Integral curves of the vector fields $e_{1}$ and $e_{2}$, passing through the point $\left(t_{0}, x_{0}\right) \in \mathbb{R}^{2}$, are determined by solutions of the ordinary differential equations

$$
\begin{aligned}
& \frac{d u}{d t}=f\left(t, x_{0}, u\right), \\
& \frac{d u}{d x}=g\left(t_{0}, x, u\right),
\end{aligned}
$$

which correspond to characteristic equations for (17). Moreover, since $d u=u_{t} d t+u_{x} d x$ on an integral surface, parameter curves, passing through a point $u\left(t_{0}, x_{0}\right)$ for $\left(t_{0}, x_{0}\right) \in \mathbb{R}^{2}$, on a solution are determined by the solutions of (25). In the subsequent section, we will define Riemannian metrics on $\delta_{0}$ and $\Sigma$ in terms of the exterior differential systems determined by the set of 1 -forms $\left\{\omega^{1}, \omega^{2}\right\}$ and $\left\{\omega^{1}, \omega^{2}, \omega^{3}\right\}$. They define local coframe fields on $\mathcal{S}_{0}$ and $\Sigma$, respectively, since both of them are linearly independent at each point on respective coordinate neighbourhoods. For detailed description of differential equations in jet bundle formalism we refer to $[29,31-$ 33].

\section{Metric Connections Associated with First-Order Differential Equations}

3.1. Connection on $\mathcal{S}_{0}$. We shall define a Riemannian metric on a coordinate neighbourhood of $\mathcal{S}_{0}$ by the sum of squares of differential 1-forms (13) as follows:

$$
g_{0}=\sum_{i} \omega^{i} \otimes \omega^{i}
$$

The coframe field $\left\{\omega^{1}, \omega^{2}\right\}$ on $\mathcal{S}_{0}$ is dual to the orthonormal frame of vector fields (14). A solution curve of (11) is attached to an integral curve of $e_{1}=\partial / \partial t+u(\partial / \partial x)$ and an integral curve of $\partial / \partial x$ to $t=$ const. By definition $g\left(e_{i}, e_{j}\right)=$ $\delta_{i j}$. It is suitable to note that Riemannian metric (26) is not uniquely defined and depends on the choice of the coframe. The coframe that we use here may be thought as "best representative" associated with a differential equation since solutions are determined by vanishing of the canonical contact form in jet bundle. Moreover, our aim is to relate 
geodesic curves of the metric connection to solution curves of given ordinary differential equation simply as $\nabla_{e_{1}} e_{1}=0$. In other words, an integral curve of (11) can be obtained as the projection of a geodesic curve.

In the formulation that we use, the one-forms $\omega^{i}, i=1,2$ are defined by the restriction or pullback of the canonical contact form $\vartheta=d x-p d t$ and $d t$ on $J^{1}(\mathbb{R}, \mathbb{R})$ to $\delta_{0}$. Since $p=u(t, x)$ on $\mathcal{S}_{0}$, in terms of a local coordinates $(t, x)$, coordinate description of the Riemannian metric is obtained as

$$
\begin{aligned}
g_{0}= & \left(1+u^{2}\right) d t \otimes d t-u(d t \otimes d x+d x \otimes d t)+d x \\
& \otimes d x
\end{aligned}
$$

by substituting $\omega^{1}=d t$ and $\omega^{2}=d x-u d t$ in (26). Notice that the components of the Riemannian metric purely depend on the right hand side of a given ODE.

The structure equations for the coframe field $\left(\omega^{1}, \omega^{2}\right)$ are determined as

$$
\begin{aligned}
& d \omega^{1}=0, \\
& d \omega^{2}=u_{x} \omega^{1} \wedge \omega^{2} .
\end{aligned}
$$

From here, we can define unique $\mathfrak{o}(2, \mathbb{R})$-valued torsion-free connection as

$$
\Theta=\left(\begin{array}{cc}
0 & -u_{x} \omega^{2} \\
u_{x} \omega^{2} & 0
\end{array}\right)
$$

In terms of $\Theta$, structure equations take the form

$$
\begin{aligned}
& d \omega^{1}=-\Theta_{2}^{1} \wedge \omega^{2}, \\
& d \omega^{2}=-\Theta_{1}^{2} \wedge \omega^{1},
\end{aligned}
$$

where $\Theta_{2}^{1}=-\Theta_{1}^{2}=-u_{x} \omega^{2}$.

Accordingly, from definition (10) of the covariant differentiation, we obtain

$$
\begin{aligned}
& \nabla_{e_{1}} e_{1}=0, \\
& \nabla_{e_{1}} e_{2}=0, \\
& \nabla_{e_{2}} e_{1}=u_{x} e_{2}, \\
& \nabla_{e_{2}} e_{2}=-u_{x} e_{1} .
\end{aligned}
$$

Then, it is concluded that 1-graph of a solution curve of (11) defines a geodesic curve of connection (29).

Curvature of the connection $\Theta$ is found as

$$
d \Theta_{1}^{2}=-\mathscr{K} \omega^{1} \wedge \omega^{2} .
$$

It is clear that $\mathscr{K}$ is exactly the Gaussian curvature of the metric compatible torsion-free connection, whose components are determined in local coordinates by the well-known formula:

$$
\Gamma_{i j}^{k}=\frac{1}{2} g^{k m}\left(\frac{\partial g_{j m}}{\partial x^{i}}+\frac{\partial g_{m i}}{\partial x^{j}}-\frac{\partial g_{i j}}{\partial x^{m}}\right) .
$$

From (32) or (33), we see that the Gaussian curvature of the metric connection is given by

$$
\mathscr{K}=-\left(u_{t}+u u_{x}\right)_{x}
$$

As a consequence, we get the following.

Theorem 1. Gaussian curvature of the metric connection (29) on the surface corresponding to the equation $d x / d t=u(t, x)$ is determined by the following inhomogeneous Burgers' equation:

$$
u_{t}+u u_{x}=-\int^{x} \mathscr{K}\left(t, x^{\prime}\right) d x^{\prime} .
$$

This representation for the curvature is worthwhile to word in some aspects: By means of expression (35), the inviscid and viscous Burgers' equations and also KdV equation can be seen as the equations for surfaces with certain Gaussian curvatures. Also, by virtue of our representation, the problem of the construction of Riemannian metrics associated with certain first-order ODEs turns out to finding a solution of certain Burgers' equations. Moreover, expression (35) for curvature is rather simple and hence it is very convenient for computational purposes in a way that it is calculated by the Lie derivative of the right hand side of a given ODE in the direction of the vector field $e_{1}=\partial_{t}+u \partial_{x}$. Besides, the spaces of constant curvature, which admit isometry group of maximal dimension, can be attached to some ODEs by solving certain inhomogeneous Burgers' equations.

From (35) it follows that $\delta_{0}$ is flat iff $u(t, x)$ satisfies

$$
u_{t}+u u_{x}=c(t)
$$

for some smooth function $c(t)$. This identity tells us that the characteristic curves of (36) correspond to the solution curves of differential equation (11). Therefore, the inviscid Burgers' equation

$$
u_{t}+u u_{x}=0
$$

corresponds to a flat surface in $J^{1}(\mathbb{R}, \mathbb{R})$ associated with the equation

$$
\frac{d x}{d t}=u(t, x) .
$$

Also, it is geometrically reasonable to investigate a question like whether the right hand side of (36) depends also on $x$, since it is ultimately related to obtaining a space of constant curvature $\mathscr{K}=0, \pm 1$ from a single ODE. These are in fact determined by the following Burgers' equations:

$$
\begin{aligned}
& u_{t}+u u_{x} \underset{(\mathscr{K}=0)}{=} c(t), \\
& u_{t}+u u_{x} \underset{(\mathscr{K}=-1)}{=} x+c(t), \\
& u_{t}+u u_{x} \underset{(\mathscr{K}=1)}{=}-x+c(t),
\end{aligned}
$$


such that the characteristic curves of these first-order PDEs correspond to solutions of the system of equations

$$
\begin{aligned}
& \frac{d x}{d t}=f(t, x), \\
& \frac{d u}{d t}=\psi(t, x),
\end{aligned}
$$

for $\psi(t, x)=c(t), \psi(t, x)=x+c(t)$, and $\psi(t, x)=-x+$ $c(t)$, respectively. Although solutions of these equations can be obtained as implicit functions of the corresponding first integrals by the method of characteristics, it is not hard to see the existence of such Riemannian metrics simply by the linear differential equation as follows:

Proposition 2. A linear first-order equation

$$
\frac{d x}{d t}+p(t) x=q(t)
$$

describes a surface of constant curvature $\mathscr{K}=0, \mp 1$ if and only if $p$ satisfies the following differential equations:

$$
\begin{aligned}
& \frac{d p}{d t}=p^{2}, \quad \text { for }(\mathscr{K}=0) \\
& \frac{d p}{d t}=p^{2} \pm 1, \quad \text { for }(\mathscr{K} \mp 1)
\end{aligned}
$$

respectively. Moreover, in the case of $\mathscr{K}=0$, if $q(t)$ is an integrating factor of (41), then $u(t, x)$ satisfies the inviscid Burgers' equation $u_{t}+u u_{x}=0$.

The proposition follows by a direct calculation by taking $u(t, x)=q(t)-p(t) x$ in (34) or (35).

On the other hand, to see what happens with our representation if the viscosity is added, identity (35) for the Gaussian curvature tells us that viscous Burgers' equation represents a surface with curvature

$$
\mathscr{K}=-v u_{x x x} .
$$

To see this, let us consider the ODE

$$
\frac{d x}{d t}=u(t, x)
$$

with $u(t, x)=-2 v(\ln \varphi)_{x}$. Then, as it is well known, $u(t, x)$ satisfies the viscous Burgers' equation

$$
u_{t}+u u_{x}=v u_{x x}, \quad v>0
$$

provided that $\varphi(t, x)$ satisfies the heat equation $\varphi_{t}=\nu \varphi_{x x}$. In this case, Gaussian curvature of the surface corresponding to (44) is determined by the differential equation

$$
u_{x x}=-\frac{1}{v} \int^{x} \mathscr{K}\left(t, x^{\prime}\right) d x^{\prime},
$$

which is equivalent to (45) due to (35). The existence of such curvature is guaranteed by the existence of a solution of the heat equation and so is (45).
Another interesting integrable model is Korteweg-de Vries $(\mathrm{KdV})$ equation

$$
u_{t}+u u_{x}+v u_{x x x}=0
$$

which admits soliton solutions and appears widely in the dynamics of shallow water waves [34]. From (35), it is seen that (47) describes a surface with Gaussian curvature

$$
\mathscr{K}=v u_{x x x x}
$$

3.2. Connection on $\Sigma$. Analogues to the previous subsection, we shall define a Riemannian metric on a coordinate neighbourhood of $\Sigma$ by the sum of squares of the elements of the coframe $\left\{\omega^{1}, \omega^{2}, \omega^{3}\right\}$ as

$$
g=\sum_{i} \omega^{i} \otimes \omega^{i}
$$

Coframe $\left\{\omega^{1}, \omega^{2}, \omega^{3}\right\}$ is dual to the orthonormal frame field of vector fields

$$
\begin{aligned}
& e_{1}=\frac{\partial}{\partial t}+f \frac{\partial}{\partial u} \\
& e_{2}=\frac{\partial}{\partial x}+g \frac{\partial}{\partial u} \\
& e_{3}=\frac{\partial}{\partial u}
\end{aligned}
$$

that is, $\omega^{i}\left(e_{j}\right)=\delta_{j}^{i}$, and by definition we have $g\left(e_{i}, e_{j}\right)=\delta_{i j}$. In terms of the local coordinates $(t, x, u)$, Riemannian metric (49) is given by

$$
\begin{aligned}
g= & \left(1+f^{2}\right) d t \otimes d t+f g(d t \otimes d x+d x \otimes d t) \\
& +\left(1+g^{2}\right) d x \otimes d x-f(d t \otimes d u+d u \otimes d t) \\
& -g(d x \otimes d u+d u \otimes d x)+d u \otimes d u .
\end{aligned}
$$

In order to construct $\mathfrak{o}(3, \mathbb{R})$-valued torsion-free connection, let us consider the structure equations for the coframe $\left\{\omega^{1}, \omega^{2}, \omega^{3}\right\}$ :

$$
\begin{aligned}
& d \omega^{1}=0, \\
& d \omega^{2}=0, \\
& d \omega^{3}=\mathscr{I} \omega^{1} \wedge \omega^{2}+f_{u} \omega^{1} \wedge \omega^{3}+g_{u} \omega^{2} \wedge \omega^{3},
\end{aligned}
$$

where $\mathscr{I}=f_{x}-g_{t}+f_{u} g-g_{u} f$. Construction of $\mathfrak{p}(3, \mathbb{R})$ valued connection needs a little bit of work. To this end, we shall solve the system of equations

$$
\begin{aligned}
\alpha \wedge \omega^{2}+\beta \wedge \omega^{3}= & 0, \\
-\alpha \wedge \omega^{1}+\gamma \wedge \omega^{3}= & 0, \\
-\beta \wedge \omega^{1}-\gamma \wedge \omega^{2}= & \mathscr{I} \omega^{1} \wedge \omega^{2}+f_{u} \omega^{1} \wedge \omega^{3} \\
& +g_{u} \omega^{2} \wedge \omega^{3} .
\end{aligned}
$$


By using Cartan's Lemma for the 1 -forms $\alpha, \beta$, and $\gamma$, we obtain

$$
\begin{aligned}
& \alpha=\frac{1}{2} \mathscr{I} \omega^{3}, \\
& \beta=\frac{1}{2} \mathscr{I} \omega^{2}+f_{u} \omega^{3}, \\
& \gamma=-\frac{1}{2} \mathscr{I} \omega^{1}+g_{u} \omega^{3} .
\end{aligned}
$$

Accordingly, we have

$$
d \omega^{i}=-\theta_{j}^{i} \wedge \omega^{j}, \quad \theta_{j}^{i}=-\theta_{i}^{j},
$$

where the connection matrix of 1-forms is defined by

$$
\theta=\left(\begin{array}{ccc}
0 & -\alpha & -\beta \\
\alpha & 0 & -\gamma \\
\beta & \gamma & 0
\end{array}\right) .
$$

Therefore, we have the following:

Theorem 3. The $\mathfrak{o}(3, \mathbb{R})$-valued torsion-free connection on $\Sigma$ is determined by

$$
\theta=\left(\begin{array}{ccc}
0 & -\alpha & -\beta \\
\alpha & 0 & -\gamma \\
\beta & \gamma & 0
\end{array}\right),
$$

where $\alpha, \beta$, and $\gamma$ are defined, respectively, by $\alpha=(1 / 2) \mathscr{I} \omega^{3}$, $\beta=(1 / 2) \mathscr{I} \omega^{2}+f_{u} \omega^{3}$, and $\gamma=-(1 / 2) \mathscr{I} \omega^{1}+g_{u} \omega^{3}$.

By definition (10) of the covariant differentiation, the covariant derivatives for the frame field $\left(e_{1}, e_{2}, e_{3}\right)$ are deduced as

$$
\begin{aligned}
& \nabla_{e_{1}} e_{1}=0, \\
& \nabla_{e_{1}} e_{2}=\mathscr{J} e_{3}, \\
& \nabla_{e_{1}} e_{3}=-\mathscr{J} e_{2}, \\
& \nabla_{e_{2}} e_{1}=-\mathscr{J} e_{3}, \\
& \nabla_{e_{2}} e_{2}=0, \\
& \nabla_{e_{2}} e_{3}=\mathscr{J} e_{1}, \\
& \nabla_{e_{3}} e_{1}=-\mathscr{J} e_{2}+f_{u} e_{3}, \\
& \nabla_{e_{3}} e_{2}=\mathscr{J} e_{1}+g_{u} e_{3}, \\
& \nabla_{e_{3}} e_{3}=-f_{u} e_{1}-g_{u} e_{2},
\end{aligned}
$$

where $\mathscr{J}=-(1 / 2) \mathscr{I}$. It follows from here that the vector fields $e_{1}$ and $e_{2}$ are covariantly constant along themselves with respect to the metric compatible connection.

As it is seen directly from (58) and (59), a PDE with one dependent and two independent variables defines codimension one distribution in three spaces. Let us denote this distribution by $\mathscr{H}$. Since $\mathscr{H}$ is spanned pointwisely by the orthonormal vector fields $\left(e_{1}, e_{2}\right)$, the curvature of $\mathscr{H}$ is determined by the equation

$$
\nabla_{e_{1}} e_{2}-\nabla_{e_{2}} e_{1}=2 \mathscr{J} e_{3}
$$

The following proposition states the intrinsic and extrinsic properties of an integral surface of (17). For the geometry of submanifolds in a Riemannian manifold, see, for instance, [35].

Proposition 4. Any integral surface of (17) is an intrinsically flat and totally geodesic submanifold of $\Sigma$.

Proof. Due to $\left[e_{1}, e_{2}\right]=-\mathscr{I} e_{3}, e_{3}$ defines a normal vector field of the integral surfaces of (17). Since $d u=u_{t} d t+u_{x} d x$ on an integral surface, Riemannian metric (51) reduces to Euclidean metric

$$
g_{u}=d t \otimes d t+d x \otimes d x,
$$

on an integral surface $u=u(t, x)$. The intrinsic curvature of the surface $u=u(t, x)$ is determined by the single independent component of Riemannian curvature tensor associated with (62). Therefore, any integral surface defines intrinsically flat surface. The second fundamental form of an integral surface identically vanishes, since on an integral surface we have $\nabla_{e_{1}} e_{3}=\nabla_{e_{2}} e_{3}=0$. It follows from here that any integral manifold of (17) is a totally geodesic submanifold of Riemannian manifold $\Sigma$.

Now, let us state and prove the theorem manifesting that the scalar curvature of the Riemannian manifold $(\Sigma, g)$ is determined by the integrability condition for (17) and the Gaussian curvatures of the surfaces corresponding to the characteristic differential equations (25).

Theorem 5. The scalar curvature of the Riemannian manifold $(\Sigma, g)$ constructed from the system of equations (17) is determined by

$$
R=-2\left(\mathscr{J}^{2}-\left(\mathscr{K}_{1}+\mathscr{K}_{2}\right)\right),
$$

where $\mathscr{J}=-(1 / 2) \mathscr{I}$ and $\mathscr{K}_{1}, \mathscr{K}_{2}$ are the Gaussian curvatures of the surfaces corresponding to equations in (25), respectively.

Proof. The proof is based on the straightforward calculation. From the second structure equations $\Omega_{j}^{i}=d \theta_{j}^{i}+\theta_{k}^{i} \wedge$ $\theta_{j}^{k}$, we determine the curvature 2 -form associated with the connection $\theta$ in terms of the coframe $\left(\omega^{1}, \omega^{2}, \omega^{3}\right)$ as

$$
\begin{aligned}
\Omega_{2}^{1}= & -3 \mathscr{J}^{2} \omega^{1} \wedge \omega^{2}+\left(\mathscr{J}_{t}+(f \mathscr{J})_{u}+f_{u} \mathscr{J}\right) \omega^{1} \wedge \omega^{3} \\
& +\left(\mathscr{J}_{x}+(g \mathscr{J})_{u}+g_{u} \mathscr{J}\right) \omega^{2} \wedge \omega^{3}, \\
\Omega_{3}^{1}= & \left(\mathscr{J}_{t}+(f \mathscr{J})_{u}+f_{u} \mathscr{J}\right) \omega^{1} \wedge \omega^{2} \\
& +\left(\mathscr{J}^{2}-\left(f_{t}+f f_{u}\right)_{u}\right) \omega^{1} \wedge \omega^{3} \\
& -\left(\mathscr{J}_{u}+f_{u x}+\left(g f_{u}\right)_{u}\right) \omega^{2} \wedge \omega^{3},
\end{aligned}
$$




$$
\begin{aligned}
\Omega_{3}^{2}= & \left(\mathscr{J}_{x}+(g \mathscr{J})_{u}+\mathscr{J} g_{u}\right) \omega^{1} \wedge \omega^{2} \\
& +\left(\mathscr{J}_{u}-g_{u t}-\left(f g_{u}\right)_{u}\right) \omega^{1} \wedge \omega^{3} \\
& +\left(\mathscr{J}^{2}-\left(g_{x}+g g_{u}\right)_{u}\right) \omega^{2} \wedge \omega^{3} .
\end{aligned}
$$

Components of the Riemann curvature tensor $\Omega_{j}^{i}=$ $\sum_{k<l} R_{j k l}^{i} \omega^{k} \wedge \omega^{l}$ are found as

$$
\begin{aligned}
& R_{212}^{1}=-3 \mathscr{J}^{2}, \\
& R_{213}^{1}=\mathscr{J}_{t}+(f \mathscr{J})_{u}+f_{u} \mathscr{J}, \\
& R_{223}^{1}=\mathscr{J}_{x}+(g \mathscr{J})_{u}+g_{u} \mathscr{J}, \\
& R_{312}^{1}=\mathscr{J}_{t}+(f \mathscr{J})_{u}+f_{u} \mathscr{J}, \\
& R_{313}^{1}=\mathscr{J}^{2}-\left(f_{t}+f f_{u}\right)_{u}, \\
& R_{323}^{1}=-\left(\mathscr{J}_{u}+f_{u x}+\left(g f_{u}\right)_{u}\right), \\
& R_{312}^{2}=\mathscr{J}_{x}+(g \mathscr{J})_{u}+\mathscr{J} g_{u}, \\
& R_{313}^{2}=\mathscr{J}_{u}-g_{u t}-\left(f g_{u}\right)_{u}, \\
& R_{323}^{2}=\mathscr{J}^{2}-\left(g_{x}+g g_{u}\right)_{u},
\end{aligned}
$$

where $R_{323}^{1}-R_{313}^{2}=0$. The sectional curvatures of the twodimensional subspaces of the tangent bundle spanned by the orthonormal pair of the vector fields $\left(e_{1}, e_{2}\right),\left(e_{1}, e_{3}\right)$, and $\left(e_{2}, e_{3}\right)$ are determined by $R_{212}^{1}, R_{313}^{1}$, and $R_{323}^{2}$, respectively. Accordingly, from (7) and (8), the scalar curvature is found by

$$
R=2\left(R_{212}^{1}+R_{313}^{1}+R_{323}^{2}\right) .
$$

It follows from here that

$$
R=-2\left(\mathscr{J}^{2}+\left(f_{t}+f f_{u}\right)_{u}+\left(g_{x}+g g_{u}\right)_{u}\right) .
$$

Using identity (34) for the Gaussian curvature of a surface corresponding to a given ODE, if we denote the Gaussian curvatures of the surfaces corresponding to equations in (25) by $\mathscr{K}_{1}$ and $\mathscr{K}_{2}$, respectively, then the theorem follows.

As a consequence of this theorem, the following are immediate:

Corollary 6. Scalar curvature of the Riemannian manifold $(\Sigma, g)$ identically vanishes if and only if $\mathscr{J}^{2}=\mathscr{K}_{1}+\mathscr{K}_{2}$.

Corollary 7. For all points on an integral manifold of (17), scalar curvature of the Riemannian manifold $(\Sigma, g)$ is determined by

$$
R=2\left(\mathscr{K}_{1}+\mathscr{K}_{2}\right) .
$$

It is also suitable to note here that a Riemannian manifold $\mathscr{M}$ is said to be flat if and only if all of the sectional curvatures identically vanish on $\mathscr{M}$. Accordingly, we have the following.
Corollary 8. Riemannian manifold $(\Sigma, g)$ is flat iff the following system of equations holds for all points of $\Sigma$ :

$$
\begin{aligned}
\mathcal{g}^{2} & =0, \\
\mathcal{J}^{2}-\left(f_{t}+f f_{u}\right)_{u} & =0, \\
\mathcal{g}^{2}-\left(g_{x}+g g_{u}\right)_{u} & =0 .
\end{aligned}
$$

It follows from this corollary that in the case of a flat manifold there exists a unique integral surface passing through each point of $\Sigma$ due to the Frobenius theorem. An integral surface is obtained by successive solutions of the system of characteristic equations in (25) determined by the concomitant Burgers' equations $f_{t}+f f_{u}=c_{1}(t)$ and $g_{x}+g g_{u}=$ $c_{2}(x)$, both of which describe intrinsically flat surfaces in $\Sigma$.

\section{Conflicts of Interest}

The authors declare that there are no conflicts of interest regarding the publication of this paper.

\section{References}

[1] T. Witelski and M. Bowen, Methods of Mathemtical Modelling Continious System and Differential Equations, Springer, Switzerland, 2015

[2] P. J. Olver, Introduction to Partial Differential Equations, Springer, Switzerland, 2014.

[3] J. M. Burgers, "A mathematical model illustrating the theory of turbulence," in Advances in Applied Mechanics, pp. 171-199, Academic Press, Inc., New York, N. Y., 1948.

[4] M. Nadjafikhah, "Lie symmetries of inviscid Burgers' equation," Advances in Applied Clifford Algebras (AACA), vol. 19, no. 1, pp. 101-112, 2009.

[5] H. Liu, J. Li, and Q. Zhang, "Lie symmetry analysis and exact explicit solutions for general Burgers' equation," Journal of Computational and Applied Mathematics, vol. 228, no. 1, pp. 1-9, 2009.

[6] I. L. Freire, "A note on 'Lie symmetries of inviscid Burgers equation,' Advances in Applied Clifford Algebras (AACA), vol. 22, no. 2, pp. 297-300, 2012.

[7] M. A. Abdulwahhab, A. H. Bokhari, A. H. Kara, and F. D. Zaman, "On the Lie point symmetry analysis and solutions of the inviscid Burgers equation," Pramana-Journal of Physics, vol. 77, no. 3, pp. 407-414, 2011.

[8] I. L. Freire, "New conservation laws for inviscid Burgers equation," Computational \& Applied Mathematics, vol. 31, no. 3, pp. 559-567, 2012.

[9] C. S. Rao and M. K. Yadav, "On the solution of a nonhomogeneous Burgers equation," International Journal of Nonlinear Science, vol. 10, no. 2, pp. 141-145, 2010.

[10] O. E. Barndorff-Nielsen and N. N. Leonenko, "Burgers' turbulence problem with linear or quadratic external potential," Journal of Applied Probability, vol. 42, no. 2, pp. 550-565, 2005.

[11] S. Kar, S. K. Banik, and D. S. Ray, "Exact solutions of Fisher and Burgers equations with finite transport memory," Journal of Physics A: Mathematical and General, vol. 36, no. 11, pp. 27712780, 2003. 
[12] S. Buyukasik and O. K. Pashaev, "Exact solutions of forced Burgers equations with time variable coefficients," Communications in Nonlinear Science and Numerical Simulation, vol. 18, no. 7, pp. 1635-1651, 2013.

[13] D. R. Nelson, "Population dynamics and Burgers' equation," Physica A: Statistical Mechanics and its Applications, vol. 274, no. 1-2, pp. 85-90, 1999.

[14] H. Liu, X. Xin, Z. Wang, and X. Liu, "Bäcklund transformation classification, integrability and exact solutions to the generalized Burgers'-KdV equation," Communications in Nonlinear Science and Numerical Simulation, vol. 44, pp. 11-18, 2017.

[15] B. Khesin and R. Wendt, The Geometry of Infinite-Dimensional Groups, Sipringer-Verlag, Berlin Heidelberg, 2009.

[16] A. Tresse, "Sur les invariants differentiels des groupes continus de transformations," Acta Mathematica, vol. 18, no. 1, pp. 1-3, 1894.

[17] M. A. Tresse, Determination des Invariantes Ponctuels de l'Equation Differentielle du Second Ordre $y^{\prime \prime}=\omega\left(x, y, y^{\prime}\right)$, Hirzel, Leipzig, 1896.

[18] S. Lie, "Classification und Integration von gewöhnlichen Differentialgleichungen zwischenxy, die eine Gruppe von Transformationen gestatten," Mathematische Annalen, vol. 32, no. 2, pp. 213-281, 1888.

[19] E. Cartan, "Sur les variétés à connexion projective," Bull. Soc. Math. France, vol. 52, pp. 205-241, 1924.

[20] N. Kamran, K. G. Lamb, and W. F. Shadwick, "The Local Equivalence Problem For $d^{2} y / d x^{2}=F(x, y, d y / d x)$ And The Painlevé Transcendents," Journal of Differential Geometry, vol. 22, no. 2, pp. 139-150, 1985.

[21] C. Grissom, G. Thompson, and G. Wilkens, "Linearization of second order ordinary differential equations via Cartan's equivalence method," Journal of Differential Equations, vol. 77, no. 1, pp. 1-15, 1989.

[22] M. E. Fels, "The equivalence problem for systems of secondorder ordinary differential equations," Proceedings of the London Mathematical Society, vol. 3-71, no. 1, pp. 221-240, 1995.

[23] M. Crampin and D. J. Saunders, "Cartan's concept of duality for second-order ordinary differential equations," Journal of Geometry and Physics, vol. 54, no. 2, pp. 146-172, 2005.

[24] S. Frittelli, C. Kozameh, and E. T. Newman, "Differential geometry from differential equations," Communications in Mathematical Physics, vol. 223, no. 2, pp. 383-408, 2001.

[25] E. T. Newman and P. Nurowski, "Projective connections associated with second-order ODEs," Classical and Quantum Gravity, vol. 20, no. 11, pp. 2325-2335, 2003.

[26] M. Crampin, E. Marnez, and W. Sarlet, "Linear connections for systems of second-order ordinary differential equations," Annales de l' I. H. P. section A, vol. 65, no. 2, pp. 223-249, 1996.

[27] M. Eastwood and V. Matveev, "Metric Connections in Projective Differential Geometry," in Symmetries and Overdetermined Systems of Partial Differential Equations, vol. 144 of IMA Vol. Math. Appl., pp. 339-350, Springer, New York, NY, USA, 2008.

[28] E. Cartan, Riemannian Geometry in an Orthogonal Frame, World Scientific Publishing Co., Singapore, 2001.

[29] T. A. Ivey and J. M. Landsberg, Cartan for Beginners: Differential Geometry via Moving Frames and Exterior Differential Systems, American Mathematical Society, Providence, RI, 2003.

[30] S. Morita, Geometry of Differential Forms, volume 201 of Translations of Mathematical Monographs, AMS Providence, RI, 2001.

[31] P. J. Vassiliou, "Introduction: Geometric Approaches to Differential Equations," in Geometric approaches to differential equations (Canberra, 1995), vol. 15 of Austral. Math. Soc. Lect. Ser., pp. 1-15, Cambridge University Press, Cambridge, UK, 2000.

[32] R. L. Bryant, P. A. Griffiths, S. S. Chern, R. B. Gardner, and H. L. Goldschmidt, Exterior Differential Systems, Springer, Berlin, Germany, 1991.

[33] D. Krupka and D. Saunders, Eds., Handbook of Global Analysis, Elsevier, Oxford, UK, 2008.

[34] D. J. Korteweg and G. de Vries, "XLI. On the change of form of long waves advancing in a rectangular canal, and on a new type of long stationary waves," Philosophical Magazine, vol. 39, no. 240, pp. 422-443, 1895.

[35] Y. Aminov, The Geometry of Submanifolds, Gordon and Breach Science Publishers, Netherlands, 2001. 


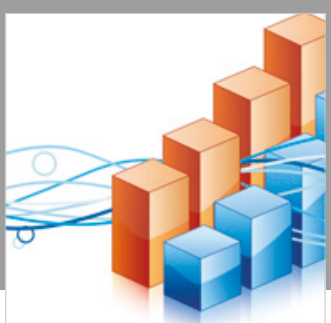

Advances in

Operations Research

\section{-n-m}
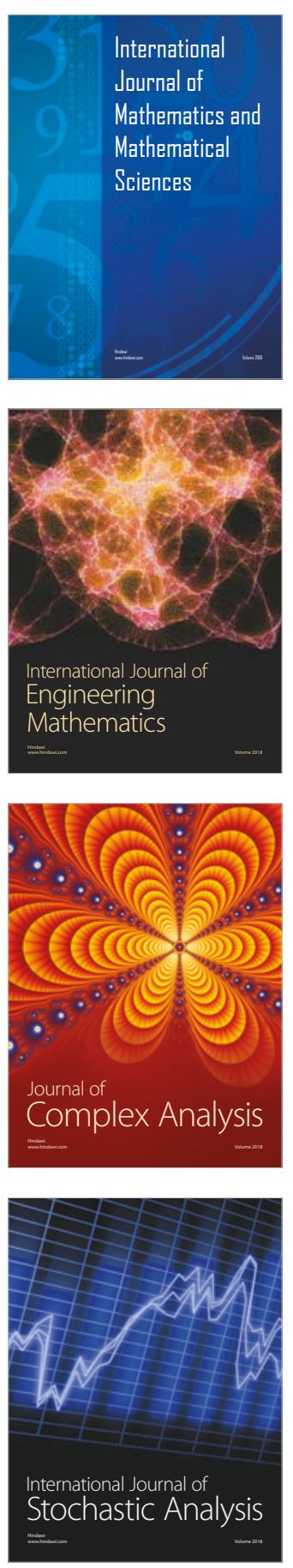
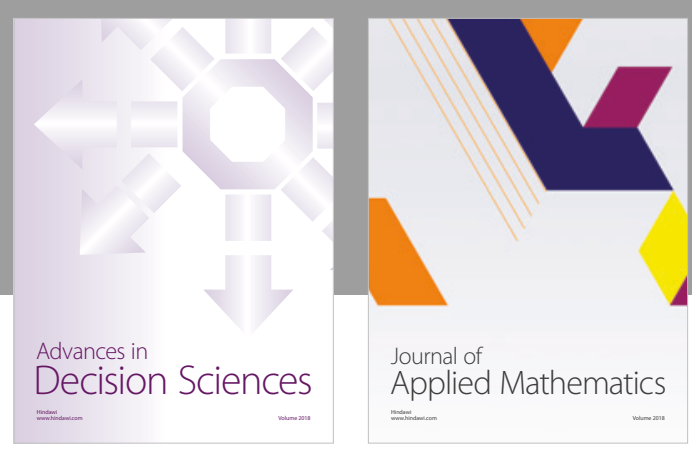

Journal of

Applied Mathematics
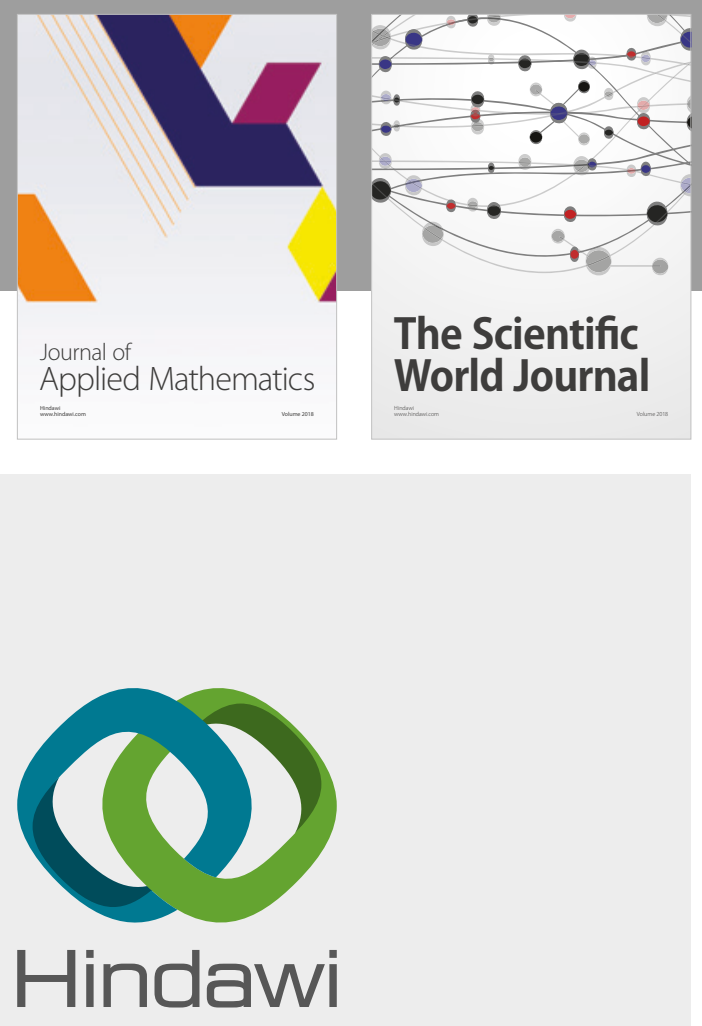

Submit your manuscripts at

www.hindawi.com

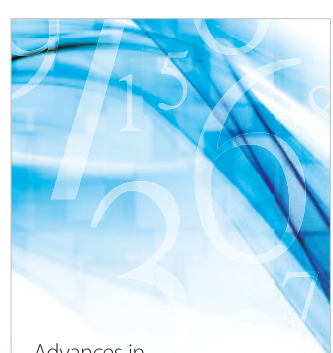

Advances in
Numerical Analysis
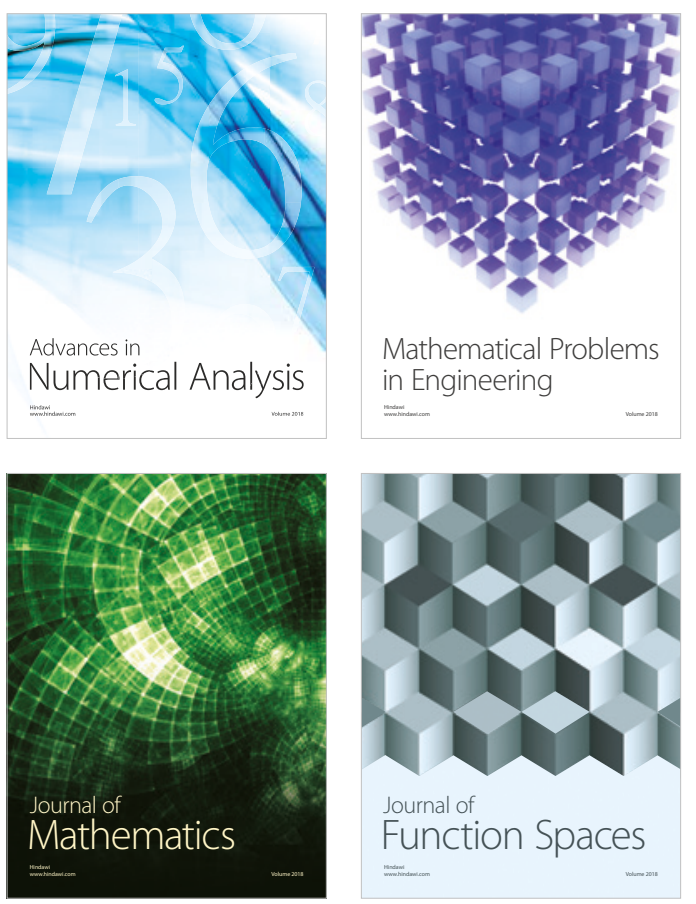

Mathematical Problems in Engineering

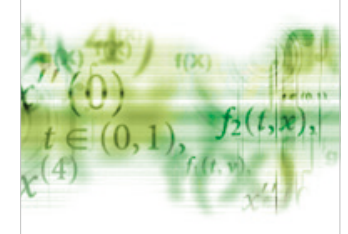

International Journal of

Differential Equations

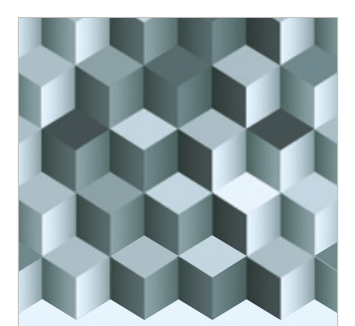

Journal of

Function Spaces

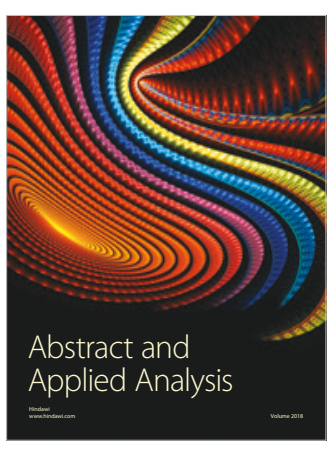

The Scientific

World Journal

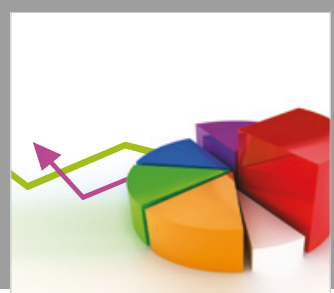

Journal of

Probability and Statistics
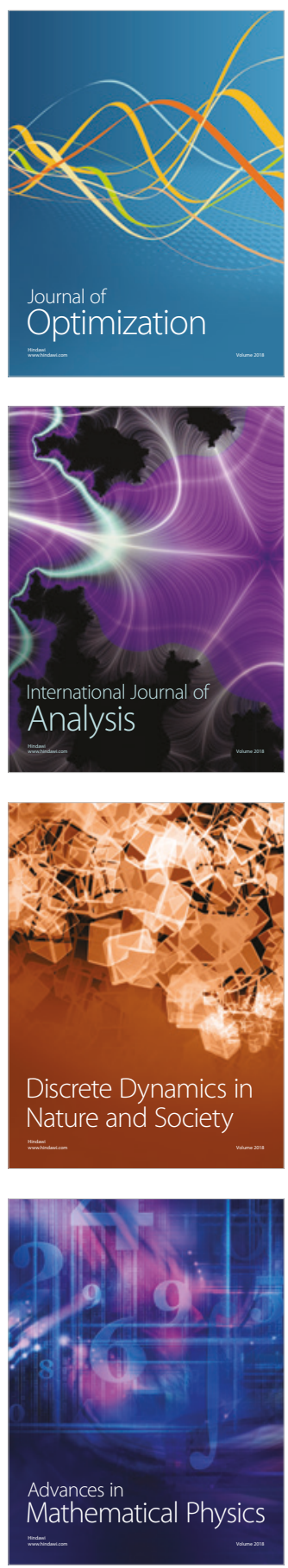